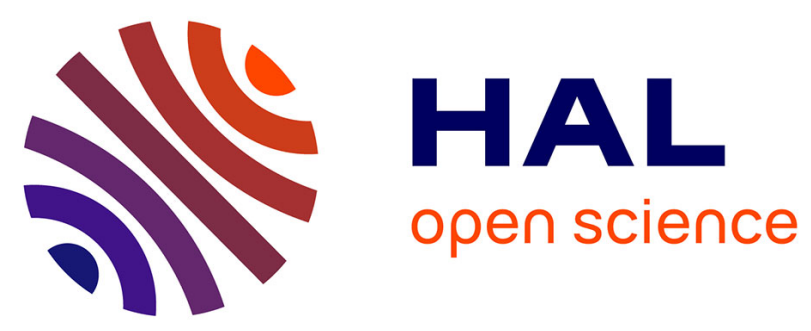

\title{
Electronic Decay of Singly Charged Ground-State Ions by Charge Transfer via van der Waals Bonds
}

\author{
Andreas Hans, Tsveta Miteva, Xaver Holzapfel, Christian Ozga, Philipp
}

Schmidt, Huda Otto, Gregor Hartmann, Clemens Richter, Nicolas Sisourat, Arno Ehresmann, et al.

\section{To cite this version:}

Andreas Hans, Tsveta Miteva, Xaver Holzapfel, Christian Ozga, Philipp Schmidt, et al.. Electronic Decay of Singly Charged Ground-State Ions by Charge Transfer via van der Waals Bonds. Physical Review Letters, 2019, 123 (21), 10.1103/PhysRevLett.123.213001 . hal-03011776

\section{HAL Id: hal-03011776 https://hal.sorbonne-universite.fr/hal-03011776}

Submitted on 18 Nov 2020

HAL is a multi-disciplinary open access archive for the deposit and dissemination of scientific research documents, whether they are published or not. The documents may come from teaching and research institutions in France or abroad, or from public or private research centers.
L'archive ouverte pluridisciplinaire HAL, est destinée au dépôt et à la diffusion de documents scientifiques de niveau recherche, publiés ou non, émanant des établissements d'enseignement et de recherche français ou étrangers, des laboratoires publics ou privés. 


\title{
Electronic Decay of Singly Charged Ground-State Ions by Charge Transfer via van der Waals Bonds
}

\author{
Andreas Hans $\odot,{ }^{1,2, *}$ Tsveta Miteva $\odot,{ }^{3}$ Xaver Holzapfel, ${ }^{1}$ Christian Ozga, ${ }^{1}$ Philipp Schmidt, ${ }^{1}$ \\ Huda Otto, ${ }^{1}$ Gregor Hartmann, ${ }^{1}$ Clemens Richter, ${ }^{4}$ Nicolas Sisourat, ${ }^{3}$ Arno Ehresmann, ${ }^{1}$ \\ Kirill Gokhberg, ${ }^{5}$ Uwe Hergenhahn, ${ }^{4,6, \dagger}$ and André Knie ${ }^{1, \$}$ \\ ${ }^{1}$ Universität Kassel, Institut für Physik und CINSaT, Heinrich-Plett-Str. 40, D-34132 Kassel, Germany \\ ${ }^{2}$ Nano and Molecular Systems Research Unit, Faculty of Science, University of Oulu, P.O. Box 3000, 90014 Oulu, Finland \\ ${ }^{3}$ Laboratoire de Chimie Physique Matière et Rayonnement, UMR 7614, Sorbonne Université, CNRS, F-75005 Paris, France \\ ${ }^{4}$ Leibniz-Institut für Oberflächenmodifizierung (IOM), Permoserstr. 15, D-04318 Leipzig, Germany \\ ${ }^{5}$ Physikalisch-Chemisches Institut, Universität Heidelberg, Im Neuenheimer Feld 229, D-69120 Heidelberg, Germany \\ ${ }^{6}$ Fritz-Haber-Institut der Max-Planck-Gesellschaft, Faradayweg 4-6, D-14195 Berlin, Germany
}

(Received 12 June 2019; published 19 November 2019)

\begin{abstract}
We report the observation of the radiative decay of singly charged noble gas ground-state ions embedded in heterogeneous van der Waals clusters. Electron-photon coincidence spectroscopy and dispersed photon spectroscopy are applied to identify the radiative charge transfer from $\mathrm{Kr}$ atoms to a $\mathrm{Ne}_{2}^{+}$dimer, which forms after single valence photoionization of $\mathrm{Ne}$ atoms at the surface of a NeKr cluster. This mechanism might be a fundamental decay process of ionized systems in an environment.
\end{abstract}

DOI: 10.1103/PhysRevLett.123.213001

Photo-induced charge transfer is among the most important mechanisms in many biochemical processes. The de- or relocalization of charge across complex organic molecules or whole biological systems accompanies or even enables a variety of photosynthetic and photocatalytic reactions $[1,2]$. While the nature of such reactions and their detailed role in biochemistry or technology may be of almost arbitrary complexity, the fundamentals of these charge transfer processes can be studied on an atomic level in prototypical and well-defined cluster systems. For weakly interacting constituents of such a cluster system, it was recently discovered that doubly charged ground-state ions can deexcite by charge transfer from their environment, even if the ion is not part of an actual chemical bond. For instance, electron transfer mediated decay (ETMD) was observed in NeKr clusters [3]. Here, a $4 p$ electron is transferred from a $\mathrm{Kr}$ atom to fill one of the two $2 p$ valence vacancies in a $\mathrm{Ne}^{2+}$ dication, and the released energy ionizes another adjacent $\mathrm{Kr}$ atom.

However, this relaxation path is energetically forbidden, if the dication is located in a homogeneous environment, as the energy excess due to the transfer of an electron from a neutral atom to a doubly charged neighbor is not sufficient for further ionization of the same species. Since the final state with two delocalized charges is still energetically favored compared to the one-site dicationic state, an electron is transferred and a photon is emitted. This process was termed radiative charge transfer (RCT) and observed indirectly in the form of missing energy in electron-ion coincidence studies on $\mathrm{Ne}$ and Ar dimers [4-6]. Recently, a direct measurement of the emitted photons after RCT in Ne clusters was achieved [7].

The preparation of the initial states of this class of charge transfer processes requires the creation of a doubly charged ground-state ion. Direct single-photon double ionization creates such states [6], but usually with a comparably low cross section [8]. Therefore, inner-shell photoionization followed by Auger decay is used in most studies [4,5,7], which requires the use of soft $\mathrm{x}$ rays.

A far more general photoionzation process is single valence ionization, for which typically photon energies from the ultraviolet (UV) to extreme ultraviolet (EUV) spectral range are sufficient. Although irradiation by photons of any energy above the ionization energy may cause valence ionization, the relaxation of the resulting ground-state ions embedded in an environment has not been investigated in detail so far.

Here, we show that singly photoionized atoms in their ionic ground state can be subject to a radiative electronic decay by charge transfer from an environment. As discussed below, such processes pave the way to measure relative ionization cross sections of condensed samples over a wide range of photon energies, which poses currently a challenging experimental task. A recently developed setup for electron-photon coincidence spectroscopy was used to unambiguously correlate valence ionization with the subsequent interatomic radiative decay in van der Waals clusters.

Singly valence ionized states in homogeneous clusters cannot decay by electron transfer between equal sites, as 
this would create indistinguishable and energetically degenerate states. If, however, a singly ionized atom is part of a heterogeneous environment, e.g., in the vicinity of another atom with lower valence ionization energy, the system can release energy by transferring an electron from the valence orbital of the neighbor to the initially ionized site releasing the surplus energy by photon emission. Because the final state of this charge transfer does not necessarily leave a signature in the electron or ion spectrum, the unambiguous identification of this process is only possible with photon spectroscopy and only the indirect observation of related processes was reported before [9]. In the present experiment, we observe radiative charge transfer after single valence ionization of $\mathrm{Ne}$ atoms in the prototypical system of heterogeneous $\mathrm{NeKr}$ clusters. This process is the response of a condensed sample to the exposure to UV and EUV radiation and therefore is fundamentally different from radiative charge transfer processes observed earlier in collisions of ions with neutral atoms $[10,11]$.

Luminescence from pure and doped condensed noble gases has also been investigated intensely before, mainly in the context of the formation and dynamics of excitonic excitations (see, e.g., Refs. [12,13] for overviews and Refs. [14-17] for NeKr). However, a radiative charge transfer process has not been reported.

To identify the proposed mechanism, an experiment was performed at the synchrotron radiation source BESSY II of the Helmholtz-Zentrum Berlin. Dispersed photon spectroscopy was applied to measure the photon emission spectrum of a heterogeneous $\mathrm{NeKr}$ cluster jet after valence ionization using the setup described in Ref. [18]. The $1 \mathrm{~m}$ normal incidence photon spectrometer was equipped with a $1 \mathrm{~mm}$ entrance slit, a grating with 600 lines per $\mathrm{mm}$ and a single photon detector with CsTe photocathode, sensitive for the spectral range from 120 to about $300 \mathrm{~nm}$ [18]. A pure $\mathrm{Ne}$ cluster jet was produced by supersonic expansion of $\mathrm{Ne}$ gas at a stagnation pressure of 3.3 bar through a conical nozzle (opening angle $\alpha=30^{\circ}$ ) of $40 \mu \mathrm{m}$ diameter, cooled to $120 \mathrm{~K}$ with liquid nitrogen. Heterogeneous $\mathrm{NeKr}$ clusters were produced by coexpansion of a mixture of $98.8 \% \mathrm{Ne}$ and $1.2 \% \mathrm{Kr}$ gas at the same conditions. Using common scaling laws [19], these conditions would result in $\mathrm{Ne}$ clusters of mean size $\langle N\rangle_{\mathrm{Ne}} \approx 16$ and $\mathrm{Kr}$ clusters of mean size $\langle N\rangle_{\mathrm{Kr}} \approx 6400$ in the case of expansion of pure $\mathrm{Ne}$ or $\mathrm{Kr}$ gas. We emphasize, however, that the final size and composition of mixed NeKr clusters cannot be determined easily since in general, this does not reflect the properties of clusters produced in coexpansion of mixed gases (see, e.g., Ref. [20]). The cluster jet was crossed with monochromatized synchrotron radiation of the UE112 PGM1 beam line. The exit slit of the beam line monochromator was set to $500 \mu \mathrm{m}$ resulting in a bandwidth of about $15 \mathrm{meV}$ at the atomic Ne ionization energy of $21.56 \mathrm{eV}$.

The UV emission intensities, integrated over the spectral region from 120 to $300 \mathrm{~nm}$, of the pure $\mathrm{Ne}$ jet and the

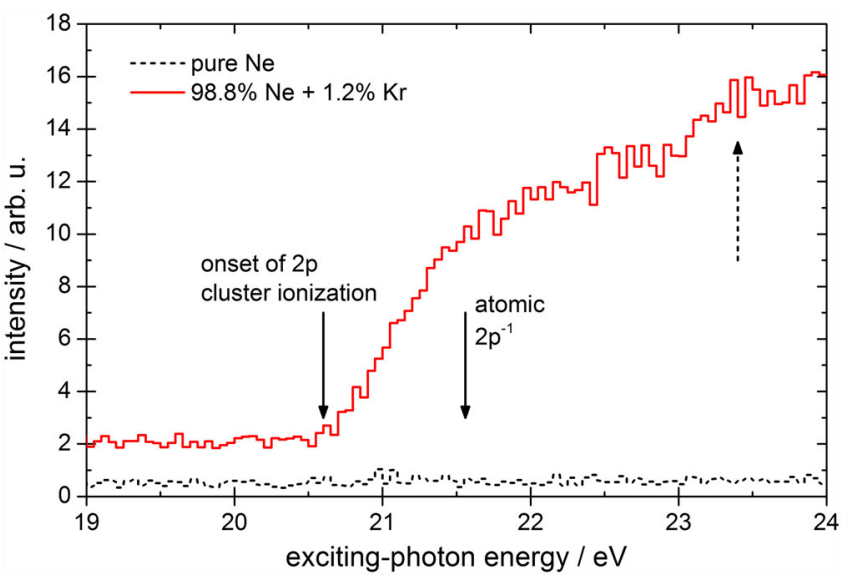

FIG. 1. UV photon excitation function of a heteronuclear $\mathrm{NeKr}$ (red solid line) and a pure Ne (black dashed line) jet, recorded in zeroth order of the photon spectrometer (i.e., integrated emission in the interval between 120 and $300 \mathrm{~nm}$ ). The atomic Ne $2 p$ valence ionization threshold and the onset of $2 p$ cluster ionization are indicated by solid arrows. The dashed arrow at $23.4 \mathrm{eV}$ indicates the photon energy at which an emission spectrum was recorded (see text).

heterogeneous $\mathrm{NeKr}$ jet as a function of the exciting-photon energy across the Ne valence ionization edge are shown in Fig. 1. The emission intensity of the pure $\mathrm{Ne}$ jet is on the detector's noise level and does not show any significant features. From pure Ne cluster jets no photon emission is expected after irradiation with photons at energies between the $2 p$ threshold and the onset of intracluster photoelectron scattering at about $36 \mathrm{eV}$ [21]. The signal from the $\mathrm{NeKr}$ jet is on a higher background level below the edge and has a steep increase at about $20.6 \mathrm{eV}$. This energy can be assigned to the $2 p$ valence ionization of small Ne clusters, which is slightly shifted to lower energies compared to atomic Ne. The shape of the NeKr curve suggests that the process leading to photon emission from the heterogeneous clusters is initiated by valence ionization of condensed Ne. The higher background level of the NeKr signal below the $\mathrm{Ne} 2 p$ edge may be attributed to higher harmonics radiation from the beam line undulator.

The underlying mechanism leading to photon emission is further elucidated by a measurement of the dispersed UV emission spectrum at a fixed exciting-photon energy of $23.4 \mathrm{eV}$. The spectrum is shown in Fig. 2 and consists of two main features peaking at about 160 and $260 \mathrm{~nm}$ (corresponding to 7.8 and $4.8 \mathrm{eV}$ ), of which the latter one is far more intense. The low-energy cutoff of the emissions cannot be identified unambiguously in the present experiment, because the sensitivity of the CsTe photocathode of the detector drastically decreases beyond $300 \mathrm{~nm}$ [18]. The resolution can be estimated from the zeroth order of the spectrometer (appearing at $0 \mathrm{~nm}$ wavelength in the emission spectrum) and is about $2 \mathrm{~nm}$. The width of the spectral features therefore is of physical origin. 


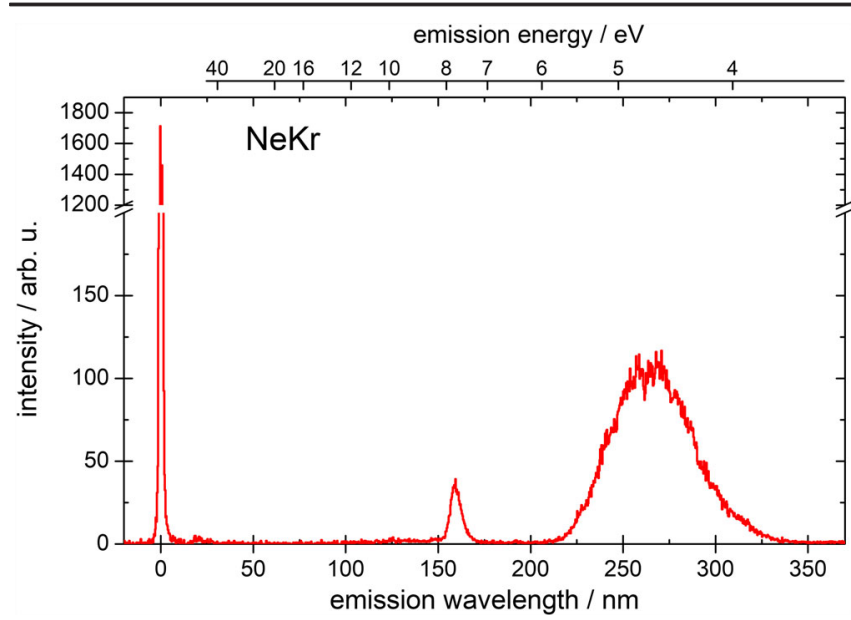

FIG. 2. Dispersed UV photon emission spectrum of a $\mathrm{NeKr}$ cluster jet excited with a photon energy of $23.4 \mathrm{eV}$. The sharp peak is the 0th order of the spectrometer, which appears at $0 \mathrm{~nm}$ and is shown as a benchmark for the spectral resolution. Note that the high-wavelength side of the feature peaking at $260 \mathrm{~nm}$ is strongly influenced by the detector efficiency which decreases strongly beyond $300 \mathrm{~nm}$ [18].

Photon emission in the range and shape of the feature at $160 \mathrm{~nm}$ has been reported before from pure $\mathrm{Kr}$ clusters after electron bombardment, and has been attributed to emission from $\mathrm{Kr}_{2}^{*}$ in neutral or ionic clusters [22], or $\left(\mathrm{Kr}_{4}^{+}\right)^{*}$ [23]. Photoelectron impact excitation of neutral $\mathrm{Kr}$ is possible at the present exciting-photon energy of $23.4 \mathrm{eV}$ [21,24,25], and a weak contribution from higher harmonics radiation from the beam line undulator, enabling the creation of excited $\mathrm{Kr}$ complexes in ionic clusters, cannot be excluded. However, the origin of this feature in the present spectrum cannot be explained with certainty.

To the contrary, no emission resembling the shape and spectral range of the second feature was reported before, indicating that these photons are emitted neither from excited $\mathrm{Ne}$ nor $\mathrm{Kr}$ atoms within the cluster or after evaporation from the cluster.

For an unambiguous attribution of the photon emission to $\mathrm{Ne}$ cluster valence ionization, we applied electronphoton coincidence spectroscopy to correlate the emitted photons with the ionization process in a separate experiment. A recently developed mirror assembly in combination with a magnetic bottle electron spectrometer as described in Ref. [26] was used to enhance the efficiency of electron-photon coincidence detection. The same detector as in the first experiment was used for photon detection. In this second experiment, a $\mathrm{NeKr}$ gas mixture with the same mixing ratio was expanded through a conical nozzle of $80 \mu \mathrm{m}$ diameter (opening angle $\alpha=30^{\circ}$ ) at a stagnation pressure of 1.2 bar, cooled to $94 \mathrm{~K}$ using liquid $\mathrm{He}$ (resulting in $\langle N\rangle_{\mathrm{Ne}} \approx 20$ and $\mathrm{Kr}$ clusters of mean size $\langle N\rangle_{\mathrm{Kr}} \approx 8800$ in the case of expansion of pure $\mathrm{Ne}$ or $\mathrm{Kr}$ gas [19]). Electron-photon coincidence spectra were obtained

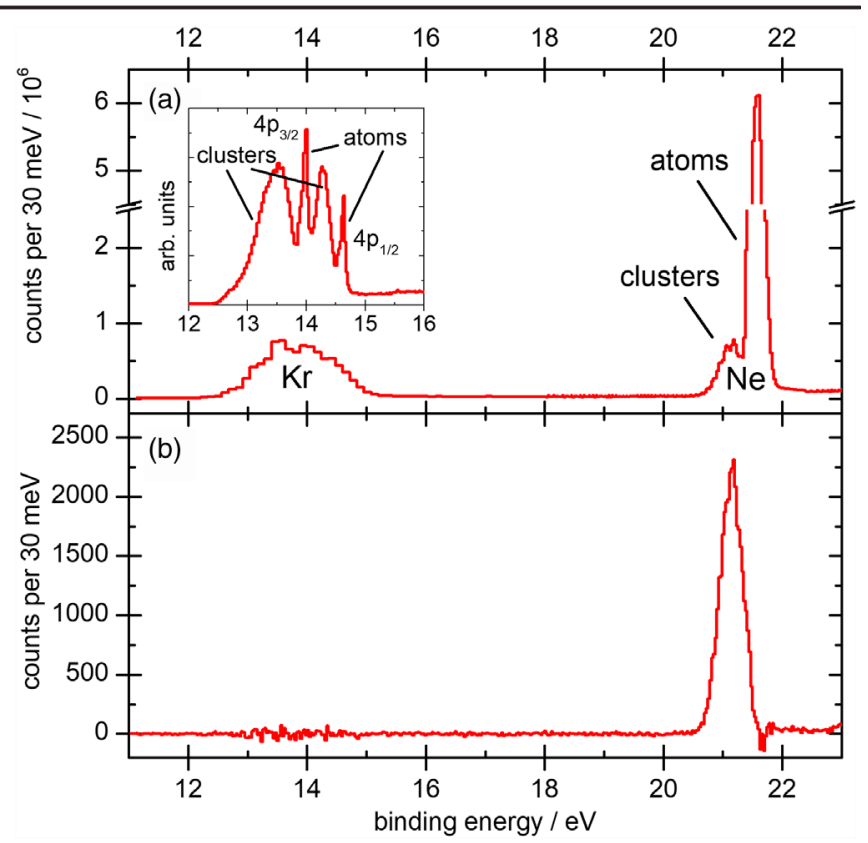

FIG. 3. Valence electron spectrum of a NeKr cluster jet, ionized at a photon energy of $23 \mathrm{eV}$. (a) The total electron spectrum shows three distinct features attributed to electrons from $\mathrm{Kr}$ and $\mathrm{Ne}$ atoms and clusters. In a high-resolution spectrum of the $\mathrm{Kr}$ feature (see inset), two $4 p$ fine structure components from $\mathrm{Kr}$ atoms and the corresponding cluster signals can be identified. (b) Electron spectrum filtered for true electron-photon coincidences.

at a photon energy of $23.0 \mathrm{eV}$ with an exit slit width of $250 \mu \mathrm{m}$.

The full valence electron spectrum of a $\mathrm{NeKr}$ cluster jet ionized at this photon energy is shown in Fig. 3(a). Features attributed to ionization of the $\mathrm{Kr} 4 p$ and $\mathrm{Ne} 2 p$ levels can be identified around an electron binding energy of 14 and $21 \mathrm{eV}$, respectively. For all atomic photoelectron lines the corresponding photoelectrons from clusters appear at slightly lower binding energy. From a high-resolution measurement of the $\mathrm{Kr} 4 p$ feature [inset in Fig. 3(a)] it can be estimated that about $85 \%$ of all $\mathrm{Kr}$ atoms in the jet are condensed in clusters. The low-energy onset of photoelectrons from condensed $\mathrm{Ne}$ at about $20.6 \mathrm{eV}$ agrees well with the observed threshold of photon emission in Fig. 1. Figure 3(b) shows the electron spectrum obtained by selecting only events in which also a photon was detected. Random coincidences of uncorrelated electrons and photons have been subtracted [26]. Only the ionization of the $2 p$ level in Ne clusters is correlated to photon emission, which confirms the suggested observation of radiative charge transfer.

The process can be investigated in more detail by comparing the observed photon emission energies in Fig. 2 with the measured electron binding energies of condensed $\mathrm{Ne}$ and $\mathrm{Kr}$ in Fig. 3. Using a simplified $\mathrm{NeKr}$ dimer model, the maximum in the emission spectrum can 
be estimated using the difference of the maxima in binding energies of $\mathrm{Ne}$ and $\mathrm{Kr}$ cluster electrons in the electron spectrum. The resulting experimental value of about $21.0-14.0 \mathrm{eV}=7.0 \mathrm{eV}$ is in significant disagreement with the observed photon spectrum.

However, it was observed before, that nuclear dynamics plays an important role after ionization of noble gas clusters [7,27]. If the subsequent relaxation is slow, which is true for the radiative decay, nuclear dynamics has to be taken into account when estimating the photon emission spectra from ionized noble gas clusters. It was observed recently for RCT to dicationic states, that the ionized site forms a dimer with a random neighbor, which additionally dissipates its vibrational energy to the surrounding before the photon is emitted [7].

Furthermore, most heterogeneous noble gas clusters are known to occur in core-shell structures with the heavier element forming the core and the lighter element arranging at the surface [28-30]. Because $\mathrm{Ne}$ is much lighter than $\mathrm{Kr}$, it is likely that an ionized $\mathrm{Ne}$ atom is at the surface of a large heterogeneous $\mathrm{NeKr}$ cluster and forms a $\mathrm{Ne}_{2}^{+}$with another $\mathrm{Ne}$ atom. Note that despite the large $\mathrm{Kr}$ core many $\mathrm{Ne}$ neighbors may be available to an ionized $\mathrm{Ne}$ atom in the surface shell. Although the exact size of the clusters and their composition is not measured in the present experiment, it is reasonable to employ a Ne dimer ion and a single adjacent $\mathrm{Kr}$ atom as a model for the charge transfer process to estimate the energetics.

We computed the ground-state potential energy curve (PEC) of $\mathrm{Ne}_{2}$ using the coupled cluster singles and doubles and perturbative triples method [CCSD(T)], and the aug-ccpVQZ basis set [31]. The PECs of the singly ionized dimer were computed with the Configuration Interaction (CI) method. The CI expansion comprises all single and double excitations from the reference configurations. The latter comprise all possible one-hole configurations in which 15 electrons occupy the 8 valence orbitals of $\mathrm{Ne}_{2}$. The aug-ccpV5Z basis set [31] was employed in the case of the singly ionized states. For all calculations, we used the MOLPRO package [32].

As illustrated in Fig. 4, only $18.6 \mathrm{eV}$ can be gained by a transition from $\mathrm{Ne}_{2}^{+}$to $\mathrm{Ne}_{2}$ at the equilibrium distance of the former (about $1.7 \AA$ ), to be compared with an ionization energy of $21.6 \mathrm{eV}$. The additional electron, which is necessary for this process, is transferred from an adjacent $\mathrm{Kr}$ atom to the $\mathrm{Ne}$ dimer. An energy of $14.0 \mathrm{eV}$ is required to release the $4 p$ electron from the $\mathrm{Kr}$ atom, resulting in the remaining $4.6 \mathrm{eV}$ for the emitted photon. This rough estimate of the emitted photon energy is close to the observed maximum in the photon spectrum. We think that the width of the photon emission feature is explained by the cluster size distribution and shifts of ionization energies in the larger clusters in the present experiment. Thus, from comparison of the experimental photon spectrum with our calculations we conclude that under the present experimental conditions a $\mathrm{Ne}_{2}^{+}$dimer forms prior to the charge

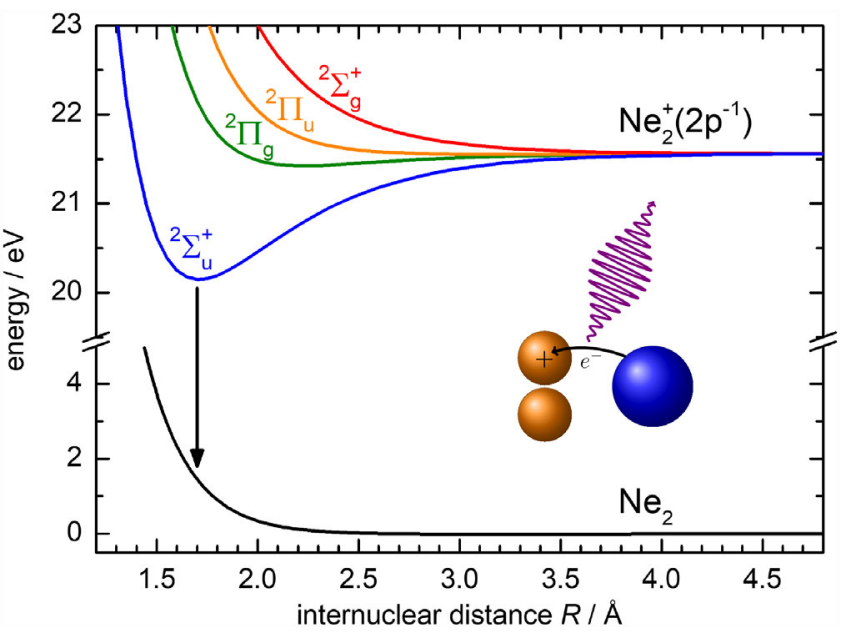

FIG. 4. Potential energy curves of the neutral and valence ionized $\mathrm{Ne}$ dimer as a function of the internuclear distance $R$. A vertical arrow marks the transition at the equilibrium distance of the $\mathrm{Ne}_{2}^{+}$dimer to the neutral dimer's ground state yielding about $18.6 \mathrm{eV}$. As illustrated, the electron required for this transition is transferred from a neighboring $\mathrm{Kr}$ atom (blue sphere) and a photon is emitted.

transfer also in heterogeneous $\mathrm{NeKr}$ clusters. The complete process can be summarized as

$$
\begin{aligned}
\mathrm{Ne}_{n} \mathrm{Kr}_{m}+h \nu & \rightarrow \mathrm{Ne}^{+}\left(2 \mathrm{p}^{-1}\right) \mathrm{Ne}_{n-1} \mathrm{Kr}_{m}+e_{\text {photo }} \\
\mathrm{Ne}^{+}\left(2 \mathrm{p}^{-1}\right) \mathrm{Ne}_{n-1} \mathrm{Kr}_{m} & \rightarrow \mathrm{Ne}_{2}^{+}\left(2 \mathrm{p}^{-1}\right) \mathrm{Ne}_{n-2} \mathrm{Kr}_{m} \\
\mathrm{Ne}_{2}^{+}\left(2 \mathrm{p}^{-1}\right) \mathrm{Ne}_{n-2} \mathrm{Kr}_{m} & \rightarrow \mathrm{Ne}_{n} \mathrm{Kr}^{+}\left(4 \mathrm{p}^{-1}\right) \mathrm{Kr}_{m-1}+h \nu_{\mathrm{RCT}}
\end{aligned}
$$

The correlation between photoionization (1) and RCT (3) is obtained by the photon-coincident electron spectrum while the dimer formation (2) is deduced from comparison of the experimental photon spectrum with theory.

We envision various applications of this new mechanism for the investigation of ionizing processes in heterogeneous clusters and at surfaces and interfaces. The measurement of ionization cross sections of condensed samples is typically challenging for common electron spectroscopy methods due to the high scattering probability of electrons in bulk matter. Furthermore, for photon energy dependent studies the transmission of the electron spectrometer used needs to be quantified carefully. Here, we present a method for measurements of relative ionization cross sections which is independent of the electron energy and unaffected by scattering processes. It is therefore applicable over a wide range of photon energies and even to the bulk of condensed samples, from where photoelectrons typically cannot escape. Using photon-electron coincidence spectroscopy, our method enables us to filter electron spectra for ionization events in a specified surrounding, e.g., at surfaces or interfaces which allow radiative charge transfer. 
In conclusion, we have observed a radiative charge transfer to singly ionized ground state ions embedded in a heterogeneous environment. Unambiguous correlation of $\mathrm{Ne}$ ionization and subsequent photon emission was confirmed by electron-photon coincidence spectroscopy on $\mathrm{NeKr}$ clusters. Evidence for dimer formation prior to the charge transfer was presented by comparing the observed photon emission energies with calculations. We assume this process to be a fundamental electronic decay mechanism in weakly bound matter and suggest different applications for the investigation of ionizing processes in clusters and at surfaces and interfaces.

We acknowledge Helmholtz-Zentrum Berlin for beam time allocation and support of the BESSY II staff during the experiments. This work was funded by the Deutsche Forschungsgemeinschaft (DFG, German Research Foundation) Projektnummer 328961117 SFB 1319 ELCH and research unit FOR 1789. X. H. acknowledges the Otto-Braun-Fonds of the University of Kassel for financial support. The project has received funding from Agence Nationale de la Recherche through the Program No. ANR16-CE29-0016-01.

*hans@physik.uni-kassel.de

${ }^{\dagger}$ Also at: Max-Planck-Institut für Plasmaphysik, Wendelsteinstr. 1, D-17491 Greifswald, Germany.

*knie@ physik.uni-kassel.de

[1] M. Bixon and J. Jortner, in Advances in Chemical Physics, edited by I. Prigogine and S. A. Rice, Advances in Chemical Physics Vol. 106 (John Wiley \& Sons, Inc, Hoboken, NJ, USA, 1999), pp. 35-202.

[2] L. Zhang, H. H. Mohamed, R. Dillert, and D. Bahnemann, J. Photochem. Photobiol. C 13, 263 (2012).

[3] D. You et al., Nat. Commun. 8, 14277 (2017).

[4] N. Saito, Y. Morishita, I. H. Suzuki, S. D. Stoychev, A. I. Kuleff, L. S. Cederbaum, X.-J. Liu, H. Fukuzawa, G. Prümper, and K. Ueda, Chem. Phys. Lett. 441, 16 (2007).

[5] K. Kreidi et al., Phys. Rev. A 78, 043422 (2008).

[6] I. Higuchi, T. Ouchi, K. Sakai, H. Fukuzawa, X.-J. Liu, K. Ueda, H. Iwayama, K. Nagaya, M. Yao, D. Ding, D. Zhang, Y. Tamenori, and N. Saito, J. Phys. Conf. Ser. 235, 012015 (2010).

[7] A. Hans, V. Stumpf, X. Holzapfel, F. Wiegandt, P. Schmidt, C. Ozga, P. Reiß, L. B. Ltaief, C. Küstner-Wetekam, T. Jahnke, A. Ehresmann, P. V. Demekhin, K. Gokhberg, and A. Knie, New J. Phys. 20, 012001 (2018).

[8] D. M. P. Holland, K. Codling, G. V. Marr, and J. B. West, J. Phys. B 12, 2465 (1979).

[9] D. Buchta, S. R. Krishnan, N. B. Brauer, M. Drabbels, P. O'Keeffe, M. Devetta, M. Di Fraia, C. Callegari, R. Richter,
M. Coreno, K. C. Prince, F. Stienkemeier, R. Moshammer, and M. Mudrich, J. Phys. Chem. A 117, 4394 (2013).

[10] R. Johnsen and M. A. Biondi, Phys. Rev. A 18, 996 (1978).

[11] J. S. Cohen and J. N. Bardsley, Phys. Rev. A 18, 1004 (1978).

[12] G. Zimmerer, in Luminescence of Inorganic Solids, edited by B. Di Bartolo, V. Goldberg, and D. Pacheco, Luminescence of Inorganic Solids Vol. 42 (Springer US, Boston, MA, 1978), , pp. 627-643.

[13] N. Schwentner, E.-E. Koch, and J. Jortner, Electronic Excitations in Condensed Rare Gases, Springer Tracts in Modern Physics Vol. 107 (Springer, Berlin, 1985).

[14] D. Pudewill, F.-J. Himpsel, V. Saile, N. Schwentner, M. Skibowski, E. E. Koch, and J. Jortner, J. Chem. Phys. 65, 5226 (1976).

[15] E. Schuberth and M. Creuzburg, Phys. Status Solidi B 90, 189 (1978).

[16] A. Kanaev, L. Museur, F. Edery, T. Laarmann, and T. Möller, Phys. Rev. B 69, 125343 (2004).

[17] R. von Pietrowski, K. von Haeften, T. Laarmann, T. Möller, L. Museur, and A. V. Kanaev, Eur. Phys. J. D 38, 323 (2006).

[18] A. Hans, P. Schmidt, C. Ozga, G. Hartmann, X. Holzapfel, A. Ehresmann, and A. Knie, Materials 11, 869 (2018).

[19] U. Buck and R. Krohne, J. Chem. Phys. 105, 5408 (1996).

[20] E. Fasshauer, M. Förstel, S. Pallmann, M. Pernpointer, and U. Hergenhahn, New J. Phys. 16, 103026 (2014).

[21] L. B. Ltaief, A. Hans, P. Schmidt, X. Holzapfel, F. Wiegandt, P. Reiss, C. Küstner-Wetekam, T. Jahnke, R. Dörner, A. Knie, and A. Ehresmann, J. Phys. B 51, 065002 (2018).

[22] É. T. Verkhovtseva, E. A. Bondarenko, and Y. S. Doronin, Low Temp. Phys. 30, 34 (2004).

[23] Y. S. Doronin, V. N. Samovarov, and E. A. Bondarenko, Low Temp. Phys. 32, 251 (2006).

[24] J. Stapelfeldt, J. Wörmer, G. Zimmerer, and T. Möller, Z. Phys. D 12, 435 (1989).

[25] T. Möller, Z. Phys. D 20, 1 (1991).

[26] A. Hans, C. Ozga, P. Schmidt, G. Hartmann, A. Nehls, P. Wenzel, C. Richter, C. Lant, X. Holzapfel, J. Viehmann, U. Hergenhahn, A. Ehresmann, and A. Knie, Rev. Sci. Instrum. 90, 093104 (2019).

[27] H. Haberland, Surf. Sci. 156, 305 (1985).

[28] M. Lundwall, H. Bergersen, A. Lindblad, G. Öhrwall, M. Tchaplyguine, S. Svensson, and O. Björneholm, Phys. Rev. A 74, 043206 (2006).

[29] M. Lundwall, W. Pokapanich, H. Bergersen, A. Lindblad, T. Rander, G. Ohrwall, M. Tchaplyguine, S. Barth, U. Hergenhahn, S. Svensson, and O. Björneholm, J. Chem. Phys. 126, 214706 (2007).

[30] T. Arion, M. Mucke, M. Förstel, A. M. Bradshaw, and U. Hergenhahn, J. Chem. Phys. 134, 074306 (2011).

[31] T. H. Dunning, J. Chem. Phys. 90, 1007 (1989).

[32] H.-J. Werner et al., MOLPRO, version 2012.1, a package of ab initio programs (2012), see http://www.molpro.net. 